\title{
Particular aspects of a pancreatic insulinoma case
}

\author{
Motoc $\mathrm{R}^{1,2}$, Albu $\mathrm{C}^{2}$, Munteanu Irina², Dirtu Oana², Motoc Crina², Tilea $\left.\right|^{3,2}$ \\ 1 Internal Medicine Clinic III, Dept. M3, Faculty of Medicine, University of Medicine and Pharmacy Tirgu Mures, Romania \\ 2 Tirgu Mures Emergency Clinical County Hospital, Tirgu Mures, Romania \\ ${ }^{3}$ Family Medicine, Dept. M3, Faculty of Medicine, University of Medicine and Pharmacy Tirgu Mures, Romania
}

With a very low incidence (1-4 cases per 1 million per year), characterized by insulin hypersecretion, independent of the glycemia control system, insulinoma is a rare endocrine tumor, clinical with accentuated neuropsychological symptoms that hampers clinical diagnosis. We present a case of a 33 years old patient with no notable personal history, active lifestyle, non-smoker, a work environment that doesn't involve professional toxicity; a remarkable family history of a brother with type 1 diabetes mellitus and grandmother with liver adenocarcinoma was noticed; in this particular case Whipple triad was strongly suggestive, gastrointestinal upper-ultrasonography endoscopy with tissue puncture as a tumor diagnostic tool was used and laparotomy was used successfully for removing the tumor, with favorable follow-up.

Keywords: insulinoma, Whipple triad, endoscopic ultrasound exploration and puncture of, surgical procedure

Received: 20 December 2014 / Accepted: 23 November 2015

\section{Introduction}

Insulinoma is commonly a functional neuroendocrine solitary tumor with sizes up to $2-3 \mathrm{~cm}$ and a very low incidence (1-3 cases per million people per year) and a maleto-female ratio of 2:3 [1-4]. Most of insulinoma (more than 99\%) appear in the pancreas (adenoma of the islet of Langerhans) and about $10 \%$ of them are malignant. In $4-7 \%$ of cases are associated with multiple endocrine neoplasia type I (MEN-I) syndrome [5]. Insulinoma can often be suspected in the presence of Whipple triad (severe hypoglycemia in $85 \%$ of patients, symptoms of hypoglycemia at the time of the low glucose level, and reversal of the symptoms after administration of glucose or carbohydrates) [6].

\section{Case report}

We present the case of a 33 year old male patient, with no notable personal history, active lifestyle, non-smoker, a work environment that doesn't involve professional toxicity, with a remarkable family history of a brother with type 1 diabetes mellitus (diagnosed at age 19) and his grandmother with liver adenocarcinoma, without detection of other clinically relevant aspects.

Apparently disease began in early October 2010 with an agitation episode (uncontrollable gestures, tremor, postural instability, impaired speech), faintness which stopped after ingestion of sweet liquid.

Given the nature of his job, involving sustained mental strain and physical inactivity, possible epilepsy was suspected and the patient was referred to neurology. After physical examination, EEG scan no suggestive data for epilepsy were found.

${ }^{*}$ Correspondence to: Cosmin Albu
On 26.10.2010 patient was found at home by caregivers with severe hypoglycemia (blood glucose capillary 29 $\mathrm{mg} \%$ ) occurred after a sustained physical effort of 10-11 hours before without eating a proper meal and quick recovery after intravenous glucose administration.

The patient was admitted on 29.10.2010 for medical evaluation with neurological symptoms (agitation, postural instability and impaired speech), recovery after ingestion of sweet liquids, normal clinical signs, but blood glucose fasting level was $50 \mathrm{mg} \%$. Abdominal ultrasound revealed gallbladder stones, a hyperechoic, enlarged head and body of pancreas and a relatively homogeneous formation, well delimited about $2.7-2.4 \mathrm{~cm}$, weakly viewed, otherwise normal relations, raising suspicion of insulinoma.

Taking into account the remarkable family medical history, patient was advised to perform a daily check-up of his blood sugar (daily occurrence of morning hypoglycemia, blood sugar levels were $45-50 \mathrm{mg} \%$ ) and then scheduled for an appointment with the diabetologist. The patient was counseled to maintain an appropriate program of dietary and lifestyle including 3 main meals and 2-3 snacks/day, supplementation of slow absorption of carbohydrates with administration of rapid absorption carbohydrates if necessary. Clinical response was promising with improvement of symptoms and increased "a jeun" glucose levels (65-75 $\mathrm{mg} \%$ ), but later on hypoglycemia reappeared. Suspecting an insulinoma and in order to exclude other endocrine diseases, blood samples were collected for endocrine tests (TSH and FT4 hormones, "a jeun" cortisol, prolactine), with normal results but low $(54 \mathrm{mg} \%)$ levels of blood sugar. Patient was subjected to a prolonged fasting for 12 hours with continuous monitoring, determining capillary blood glucose every 90 minutes and taking venous blood sampling at glucose levels of $40 \mathrm{mg} \%$. After about 8 hours, he became incoherent with capillary blood glucose of 42 $\mathrm{mg} \%$. First a venous blood sample was taken from which 
lycaemia and insulinemia were measured. After 5 minutes another 2 similar samples were collected. Due to his worsening confusional state, agitation, intravenous glucose was administered and his state rapidly improves. The results revealed a fasting glucose/insulin ratio of 1.3. An abdominal contrast-enhanced computed tomography scan showed a $19 \mathrm{~mm}$ hypodense, well-defined boundaries and poorly -iodine tumor situated at the level of the pancreatic tail. No evidence of lymphadenopathy or distant metastasis was identified (Figure 1).

An endoscopic ultrasound-guided fine-needle aspiration biopsy of the tumor was performed revealing a hypodense tumor of about $2 \mathrm{~cm}$ diameter with a cystic component at the level of the tail of the pancreas and a hypovascular soft elastographic aspect, both before and after injection of the contrast media, emphasizing peritumoral Doppler signal and multiple images of the gallbladder stones, most

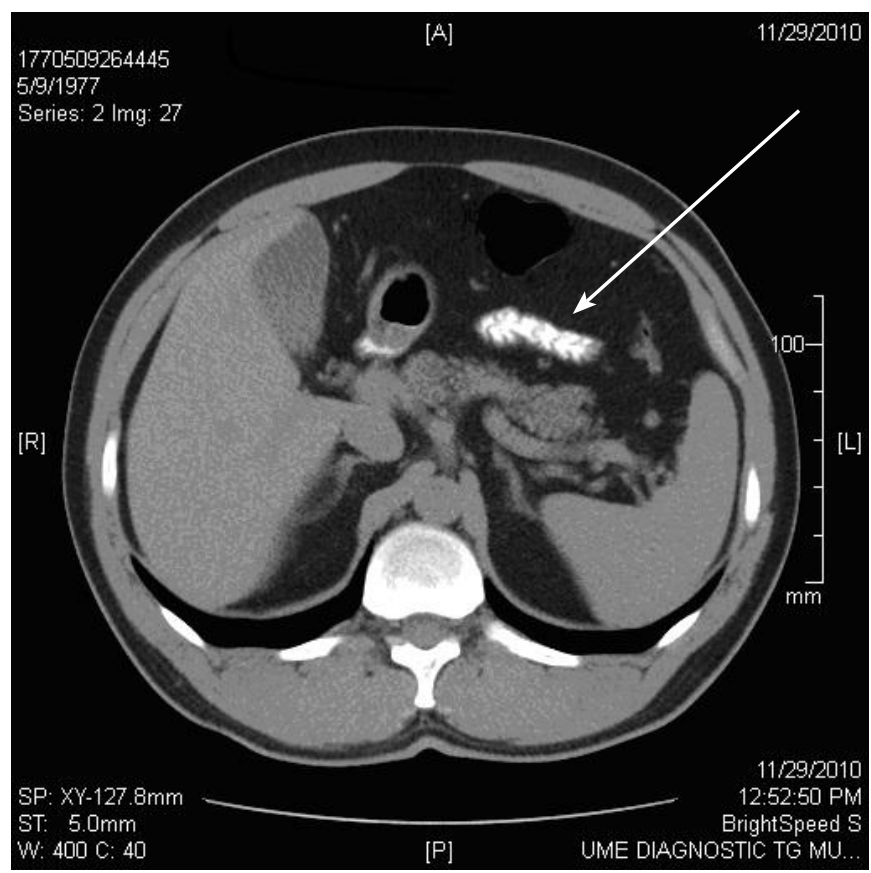

Fig. 1. Abdominal contrast-enhanced computed tomography. Localization of the insulinoma (arrow)

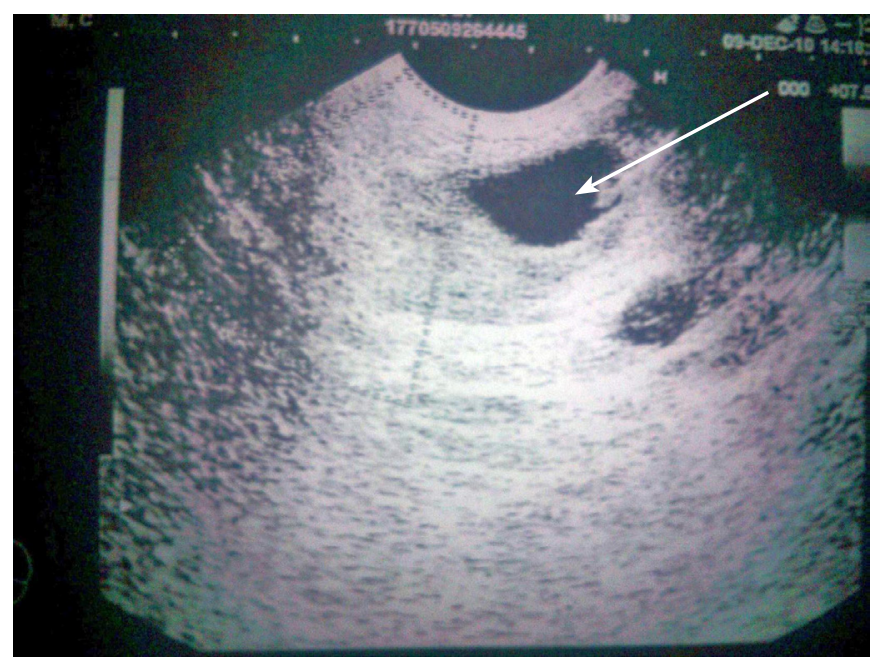

Fig. 2. Endoscopic ultrasound examination. Cystic component at the level of the tail of the pancreas (arrow) of them about $2 \mathrm{~cm}$ in diameter (figure 2). A puncture of both cystic fluid component and solid component of the tumor (in three passages) was performed, without immediate complications.

Histological examinations of the cystic component revealed a series of smears, frequent lymphocytes, rare macrophages, rare groups of cylindrical epithelial cells, some with hypertrophic nuclei, rare polymorphonuclear leukocytes and frequent erythrocytes. The solid component analysis reveals smears with hyper cellularity seen frequently represented by oval cell groups, some pseudorosettes aspects, other with a papillary dispersion with moderate nuclear dyskariosis, and in most cases with a fine granular cytoplasm and rare mitoses atypical bare dyskariotic nuclei, polymorphonuclear leukocytes and many red blood cells.

\section{Treatment and outcome}

Subsequently, considering all the data, patient was scheduled for laparotomy; a corporal-caudal pancreatic-splenectomy, cholecystectomy and drainage of omental sac were performed. The postoperative evolution of the patient was favorable, resuming orally food on the third day postoperative, being discharged with recommendation to avoid physical exertion and local trauma, with dietary regimen, tricyclic antihistamines, antithrombotic regimen, electrolytes and platelet count monitoring and fasting glucose check-up regularly or on need.

The histopathological report showed a well-differentiated endocrine tumor of uncertain behavior pT1, peripancreatic lymph nodes presenting two sinus histiocytosis, Ki67 staining positive in about 10\% of tumor nuclei, the existence of positive microscopic malign nucleus rate reaching $20 \%$.

As particular features of this case we mention young age (33 years old), abrupt and non-specific onset of symptoms, diagnostic algorithm (including the use of upper- ultrasonography gastrointestinal endoscopy with puncture) and medical treatment combined with classic laparotomy.

The outcome was favorable, at one year till now, without recurrence symptoms of insulinoma, normal abdominal CT scan.

\section{Discussions}

Insulinomas, solitary endocrine tumors, are derived from the beta cells of pancreas; with a very low incidence of 1-4 cases / million patients / year are generally benign, but with sizes over $2 \mathrm{~cm}$, have increased malignant potential [4,7]. The incidence of insulinoma is higher in autopsy studies, suggesting that these tumors frequently remain undiagnosed [8]. Insulinomas can be often suspected using the Whipple triad [9]. Most cases are women, 10\% are malignant [10]. Our patient displayed several characteristicstypical for insulinoma.

Although the technique has evolved a lot in recent years, there is still a very difficult to diagnose a positive islet. There is of course progress in the pre- and intraoperative 
diagnosis (CT, MRI, endoscopic ultrasonography intraoperatively) that allow localization and excision resection of pancreatic islet avoiding blind surgery. The combination of biphasic thin section helical CT and endoscopic ultrasonography has an almost $100 \%$ sensitivity in localizing insulinomas [11].

As treatment of insulinoma it is recommended early surgery thus preventing possible complications such as hypoglycemic encephalopathy; the recurrence is about $8 \%$ for benign insulinomas. Laparoscopic enucleation of insulinoma seems to be a feasible and safe approach and resulted in a short hospitalization and rapid recovery for most patients [12]. Pancreatectomy or pancreatoduodenectomy (Whipple's procedure) is reserved for large potentially malignant tumors or failed enucleation; it has a higher complication rate $[13,14]$. Postoperative patient monitoring is required, avoiding possible complications.

Medical treatment can be adviced for a subgroup of patients who are unable or refuse to undergo surgical treatment, for preoperative control of blood glucose levels or for unresectable metastatic disease $[15,16]$.

The case was presented with the need to attract attention crossing a therapeutic algorithm in the eco-point upper gastrointestinal endoscopy and abdominal CT scan tumor to be on the forefront for the diagnosis of insulinoma in the context of clinical evocative symptoms, with a degree of suspicion even in biological partially relevant data.

\section{Learning point}

Insulinoma must be considered in the differential diagnosis of any young patient presenting with autonomic nervous system and hypoglycemic symptoms. Diagnosis and treatment approach required for insulinomas needs interdisciplinary collaboration between internal medicine, diabetology, gastroenterology, medical imaging and of course surgery specialists.

\section{Conflicts of interest}

Authors declare no conflicts of interest that could be influenced the present case report.

\section{References}

1. Yao MM, Reynertson $\mathrm{RH}$. Insulinoma: A Case Report and Review of the Clinical Features and Diagnosis. Gundersen Lutheran Medical Journal 2005;3(2):73-6

2. Mittendorf EA, Liu YC, McHenry CR. Giant insulinoma: case report and review of the literature. J Clin Endocrinol Metab 2005;90(1):575-80

3. Andronesi D, Andronesi A, Andrei S, Tonea A, Popescu I. Etiopathogeny of The Insulinoma. Ann Fundeni Hosp 2008; 13(1-2):51-57

4. Tucker ON, Crotty PL, Conlon KC. The management of insulinoma. $\mathrm{Br} J$ Surg 2006; 93(3):264-75

5. Hart SP, Frier BM. Causes, management and morbidity of acute hypoglycaemia in adults requiring hospital admission. QJM 1998;91(7):505-10

6. Whipple AO, Frantz VK. Adenoma of islet cells with hyperinsulinism: a review. Ann Surg. 1935; 101(6):1299-1335

7. Abbasakoor NO, Healy ML, O'Shea D, et al. Metastatic insulinoma in a patient with type 2 diabetes mellitus: case report and review of the literature. Int J Endocrinol. 2011;2011:124078

8. Kimura W, Kuroda A, Morioka Y. Clinical pathology of endocrine tumors of the pancreas. Analysis of autopsy cases. Dig Dis Sci 1991;36(7):93342

9. Hirshberg B, Livi A, Bartlett DL, et al. Forty-eight-hour fast: the diagnostic test for insulinoma. J Clin Endocrinol Metab. 2000;85(9):3222-6

10. Halfdanarson TR, Rubin J, Farnell MB, et al. Pancreatic endocrine neoplasms: epidemiology and prognosis of pancreatic endocrine tumors. Endocr Relat Cancer 2008;15:409-427

11. Vaidakis D, Karoubalis J, Pappa T, et al. Pancreatic insulinoma: current issues and trends. Hepatobiliary Pancreat Dis Int. 2010;9(3):234-41

12. Karaliotas C, Sgourakis G. Laparoscopic versus open enucleation of solitary insulinoma in the body and tail of the pancreas. J Gastrointest Surgery 2009 13(10):1869.

13. Grant CS. Insulinoma. Best Pract Res Clin Gastroenterol. 2005;19(5):78398

14. Thompson NW, Czako PF, Fritts LL, et al. Role of endoscopic ultrasonography in the localization of insulinomas and gastrinomas. Surgery 1994;116:1131-38

15. de HerderWW, Niederle B, Scoazec JY, et al. Well-differentiated pancreatic tumor/carcinoma: insulinoma. Neuroendocrinology 2006;84(3):183-88

16. Cohen MS, Bower RH, Fidler SM, et al. Inhibition of insulin release by diphenylhydantoin and diazoxide in a patient with benign insulinoma. Lancet 1973; 1(7793):40-1 